\title{
IRON-BASED ALLOY FOR PLASMA-POWDER SURFACING OF SCREW CONVEYORS OF EXTRUDERS AND INJECTION MOLDING MACHINES
}

\author{
A.I. SOM \\ Plasma-Master Ltd \\ 3 Krzhizhanovsky Str., 03680, Kiev, Ukraine. E-mail: info@plasma-master.com
}

\begin{abstract}
The structure and properties of wear- and corrosion-resistant alloy of $\mathrm{Fe}-\mathrm{Cr}-\mathrm{V}-\mathrm{Mo}-\mathrm{Ni}-\mathrm{C}$ system were investigated for plasma surfacing of screw conveyors of extruders and injection molding machines. It was shown that combination of high wear resistance and ductility of the alloy is achieved due to formation of a large amount of primary vanadium carbides VC and eutectics, located in the viscous austenitic-martensitic matrix in the form of single colonies. The alloy has an excellent formation and high resistance of the deposited bead to crack formation in surfacing, that allows surfacing rather massive parts without preheating. The service life of screw conveyors deposited using this alloy is $3-5$ times higher than that of nitrated screws. 10 Ref., 2 Tables, 10 Figures.
\end{abstract}

Ke ywords : plasma-powder surfacing, alloy structure, carbides, carbide eutectic, wear resistance, coefficient of linear expansion, bead formation

To increase the service life of screw conveyors of extruders and injection molding machines the plasma-powder surfacing of working surfaces using wear-resistant alloys is widely applied in the world [1-5]. Most often for these purposes the alloys are used based on nickel and cobalt. To avoid cracks in the deposited layer the parts are preheated to 400 $500{ }^{\circ} \mathrm{C}$, and sometimes the concurrent heating is used.

The experience shows that preheating of such parts not only complicates the process of surfacing, but also significantly raises its cost. In addition, the widespread use of nickel and cobalt alloys for surfacing of screw conveyors is rather traditional than it is caused by the necessity. Although such unique properties as heat resistance, hot hardness, corrosion resistance and others are important in the operation conditions of screw extruders, however they are not decisive. Therefore, according to the author opinion, they can be successfully replaced for the cheaper and more wear-resistant alloys based on iron.

Unfortunately, the known commercial alloys based on iron have the same serious technological disadvantage as nickel or cobalt alloys, namely increased tendency to crack formation during surfacing. There is an experience of application of high-speed steel 10R6M5 for the surfacing of screw conveyors, which under the certain thermal cycle due to the effect of martensite transformation can be deposited without cracking [6]. However, as was demonstrated by industrial tests, due to insufficient ductility of this steel during operation the cracks may be formed in the deposited layer and cause cleavages, that is unacceptable.

Specially for plasma-powder surfacing of screw conveyors, the author of this article together with (c) A.I. SOM, 2016 other authors developed a new wear-resistant alloy of system $\mathrm{Fe}-\mathrm{Cr}-\mathrm{V}-\mathrm{Mo}-\mathrm{C}$ resistant to cracks [7]. It belongs to the class of high-vanadium cast irons. During its development the maximum operating conditions of these components and their structural features were taken into account.

In the present work for the first time the properties of the alloy for surfacing of screw conveyors, and experience of its industrial operation are described in detail.

Structure and phase composition of the alloy after surfacing. The microstructure and phase composition of the deposited metal was investigated by optical and electron metallography, as well as using the methods of X-ray diffraction, X-ray spectral and chemical phase microanalyses (the investigations were carried out at PWI).

The structure of metal in the initial state after surfacing consists of a solid solution, based on iron, carbide eutectic and excessive vanadium carbides VC. The characteristic feature of the structure is a very fine grain $(10-15 \mu \mathrm{m})$ and eutectic of a fan shape, which are located in the form of separate colonies as if embedded into the matrix (Figure 1, $a$ ). The shape and structure of eutectic colonies are clearly visible in the SEM-photo (Figure 1,b), produced by microscope JSM-35, where thin plates of eutectic carbides are alternated with a solid solution filling the gaps between them. Such a structure of eutectic provides the alloy with a combination of high strength and ductility at the level of $\sigma_{\mathrm{t}}=1000 \mathrm{MPa}$ and $a_{\mathrm{n}}=25 \mathrm{~kg} / \mathrm{cm}^{2}$.

The vanadium carbides are fine (up to $5 \mu \mathrm{m}$ ), have a rounded shape, and are comparatively uniformly distributed throughout the whole volume of the alloy. The total amount of carbide phase, according to the data of chemical phase analysis, is about $16 \%$. As 


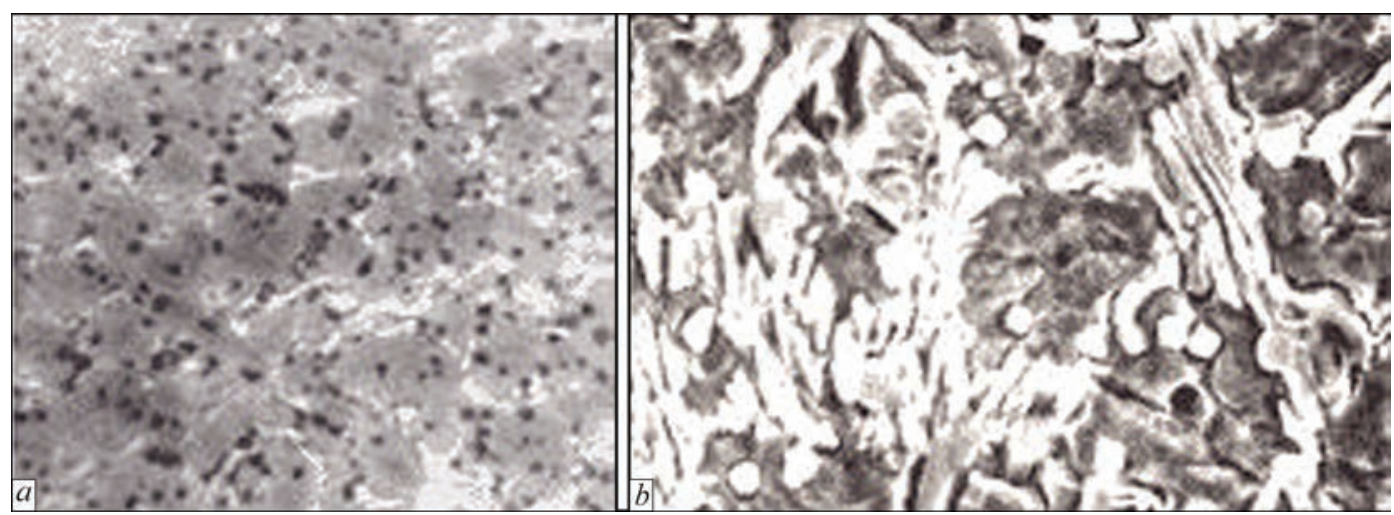

Figure 1. Microstructure of deposited metal: $a$ - optic microscope $(\times 500) ; b$ - electron microscope $(\times 3000)$

was shown by X-ray structural analysis of anode precipitation, except of vanadium carbides, the composition of carbide phase VC includes eutectic carbides, based on chromium of $\mathrm{Me}_{7} \mathrm{C}_{3}$ type, molybdenum of $\mathrm{Me}_{2} \mathrm{C}$ type and carbides of cementite type $\mathrm{Me}_{3} \mathrm{C}$, where a part of iron atoms is replaced by atoms of other elements. The matrix of the alloy represents $(\gamma+$ $\alpha$ )-solid solution with microhardness HV25-400-450. According to the data of X-ray microanalysis, the solid solution contains about, $\%: 15 \mathrm{Cr}, 3 \mathrm{~V}, 2 \mathrm{Mo}, 5 \mathrm{Ni}$.

A high degree of alloying of the solid solution with the given elements significantly delays $\gamma \rightarrow \alpha$ transformations. As is shown by investigations performed at a high-speed dilatometer under the continuous cooling from high temperatures (Figure 2), the starting point of martensitic transformation $\mathrm{M}_{\mathrm{s}}$, depending on the cooling rate, lies at the level of $110-130^{\circ} \mathrm{C}$.

Due to this fact, up to $60-80 \%$ of residual austenite is fixed in the alloy. It causes a beneficial effect to the ductility of the alloy and allows relaxing a significant part of stresses arising during rapid cooling at surfacing process.

Structure and phase composition of the alloy after heat treatment. In manufacture of screw conveyors the obligatory technological operation is high tempering, therefore, it is important to know its effect on the structure and properties of the deposited

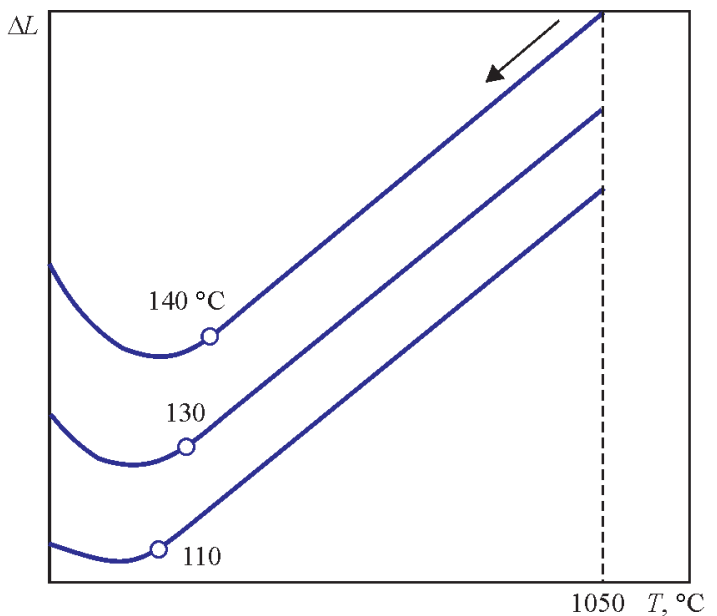

Figure 2. Dilatometric curves at continuous cooling of deposited metal from high temperatures metal. The investigations carried out in the vacuum differential Chevenar dilatometer, provide insight on the structural sensitivity of the alloy to the subsequent heating and cooling.

As is seen from the dilatometric curve (Figure 3) the phase $\gamma \leftrightarrow \alpha$ transformation occur in the deposited metal. The critical points are expressed quite clearly: $A_{c 1}$ lies at the level of $650{ }^{\circ} \mathrm{C}, A_{c 3}$ is at $850^{\circ} \mathrm{C}$.

During cooling the martensitic transformation with a significant volume effect occurs. It should be noted that structural transformations in the metal, characterized by reduction in volume (the curve is bent downwards), begin a little earlier, approximately at $550{ }^{\circ} \mathrm{C}$. At these temperatures the precipitation of fine-dispersed chromium, molybdenum and vanadium carbides from supersaturated austenite begins.

The abovementioned is confirmed by dilatograms of tempering at these temperatures (Figure 4), indicating a significant volume effect of additional martensite transformation, which occurs in the alloy due to depletion of austenite with the alloying elements and reduction of its resistance to decay. The HRC hardness of the deposited metal at the same time increases from 43-44 to 52-53 (Figure 5). Its maximum value is achieved at the tempering temperature of $650{ }^{\circ} \mathrm{C}$. Also at the same temperature the maximum decay of austenite is observed. The increase in duration of ex-

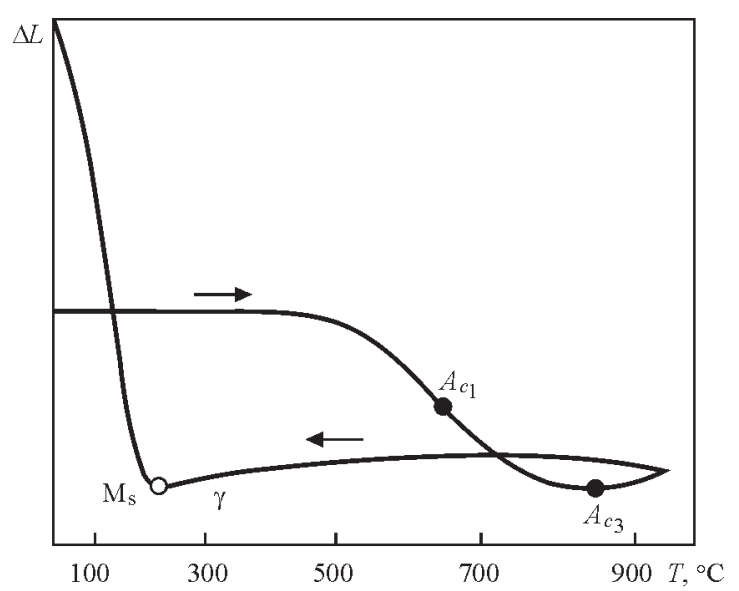

Figure 3. Dilatometric curve at continuous heating and cooling of deposited metal at $150-170{ }^{\circ} \mathrm{C}$ 


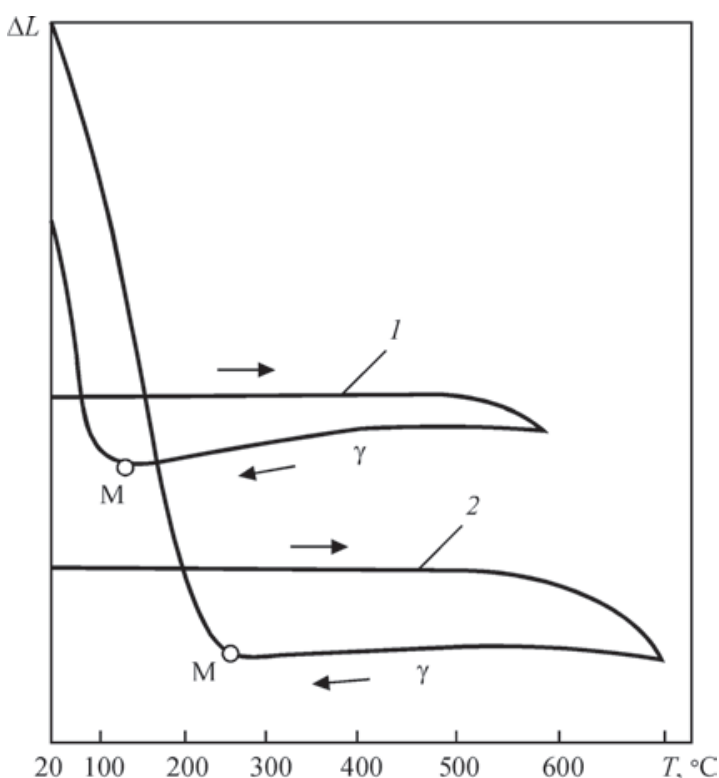

Figure 4. Dilatometric curves of tempering of deposited metal: $1-600^{\circ} \mathrm{C}, 1 \mathrm{~h} ; 2-700{ }^{\circ} \mathrm{C}, 1 \mathrm{~h}$

posure does not cause a noticeable increment in hardness since the most intensive precipitation of carbides is apparently occurs at the initial period.

After tempering at $700{ }^{\circ} \mathrm{C}$, due to a greater depletion of austenite, the volume effect of martensite transformation is expressed more clearly (see Figure 4 , curve 2), although the hardness of deposited metal the is somewhat lower (see Figure 5). Here the reduction in hardness, as compared to tempering at $650{ }^{\circ} \mathrm{C}$, occurs apparently due to decomposition of the initial martensite and carbides coagulation.

The precipitation of dispersed carbides at tempering of the alloy was managed to be revealed by SEM investigations. In Figure 6 the typical places of precipitation of these carbides are shown. They are originated both in the grains of solid solution, as well as in eutectic colonies, apparently in those places where the most favorable thermodynamic conditions for their formation exist, like dislocations in martensite, phase boundaries, etc.

Service properties of deposited metal. Taking into account structural sensitivity of deposited metal to heat treatment, let us consider the influence of tempering on its wear resistance and mechanical properties.

Wear resistance. The tests were carried out in laboratory installation NK-M [8]. A fine-dispersed quartz sand with grain size of $\leq 80 \mu \mathrm{m}$ served as abrasive. As a reference the specimens of annealed steel 45 were used.

As is seen from Figure 5, at first the wear resistance of deposited metal grows to $400{ }^{\circ} \mathrm{C}$ in the range of tempering temperatures and then slightly decreases. It is connected, first of all, with the influence of tempering on the amount and stability of residual austenite in the alloy, which plays a large role in wear processes $[9,10]$. The positive role of metastable austenite con-

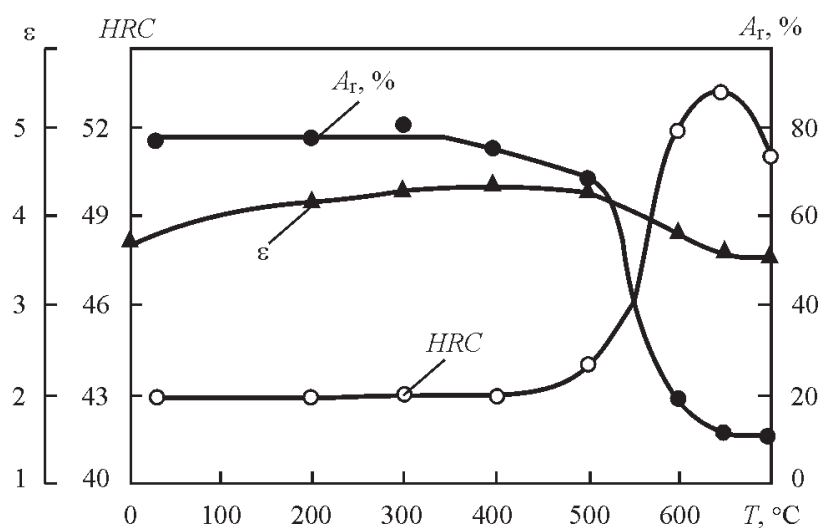

Figure 5. Influence of tempering temperature on hardness $H R C$, wear resistance $\varepsilon$ and amount of residual austenite $A_{\mathrm{r}}$ sists in the fact that not only properly itself is a good resistant to abrasive wear, but also firmly preserves the fine vanadium carbides against spalling during friction due to formation of martensite deformation in the surface layer. After low and medium tempering the total amount of residual austenite is still retained, but as a result of diffusion redistribution of carbon its stability decreases and more favorable conditions for formation of deformation martensite are created, by which the observed increase in wear resistance may be explained.

High tempering, especially at $650{ }^{\circ} \mathrm{C}$, causing decay of residual austenite, causes a somewhat reduction of wear resistance of the alloy as compared to medium tempering, though the hardness in this case is much higher.

The additional wear resistance of the deposited metal is given by nitration, which, as a rule, is applied to protect the rest part of the screw conveyor from wear in manufacture of new parts. Due to high alloying of the metal, a nitrated layer is produced not deep (up to $0.05 \mathrm{~mm}$ ), but very hard (HV25-1288 (Figure 7)). Due to this, the wear process of the deposited metal, especially at the initial stage, is significantly delayed.

Figure 8 shows the results of comparative tests in the same conditions of the considered alloy and some typical representatives of wear-resistant Ni-, Co-, Fe-

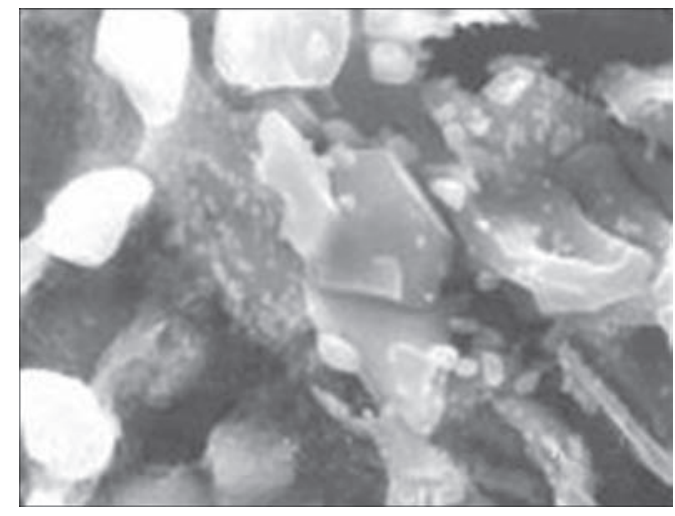

Figure 6. Precipitation of thin-dispersed carbides in the alloy after tempering at $650{ }^{\circ} \mathrm{C}$ for $1 \mathrm{~h}$ 


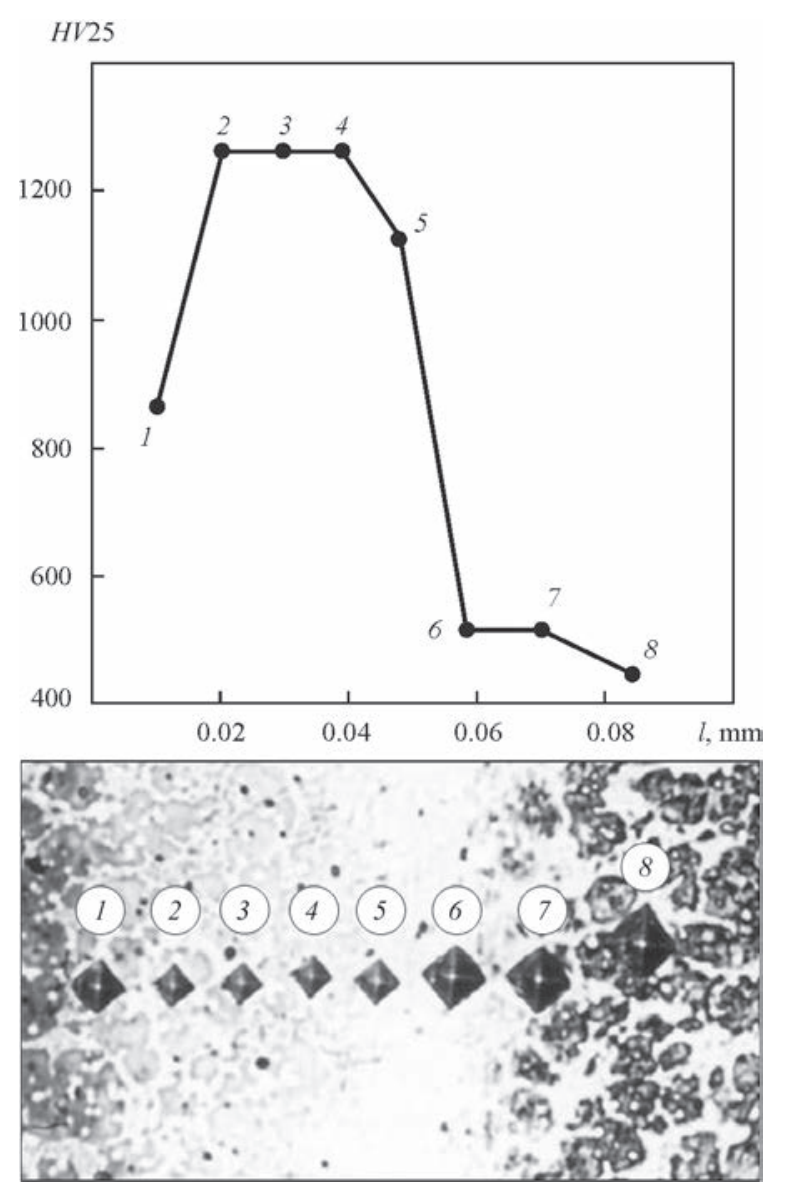

Figure 7. Distribution of hardness in nitrated layer of deposited metal

based alloys. The chemical composition of the tested alloys is given in Table 1 .

As is seen from Figure 8, the relative wear resistance of the new alloy both in the initial state as well as after tempering with nitration is considerably higher than that of nickel (PG-SR4) and cobalt (Stellite 6) alloys. After nitration, it also becomes higher than that of alloy Sormite 1.

Coefficient of linear expansion. CLE is an important characteristic for evaluation of operability of deposited product under the conditions of elevated temperatures. The closer are the CLE values of the deposited and base metals, the lower the level of residual stresses, the lower deformation of the deposited part and, finally, the better operating conditions of bimetallic part.

The results of measuring the CLE of the considered alloy at different temperatures are given in Table 2. For comparison, the data for steel $40 \mathrm{KhN}$ used

Table 1. Chemical composition and hardness of alloys tested

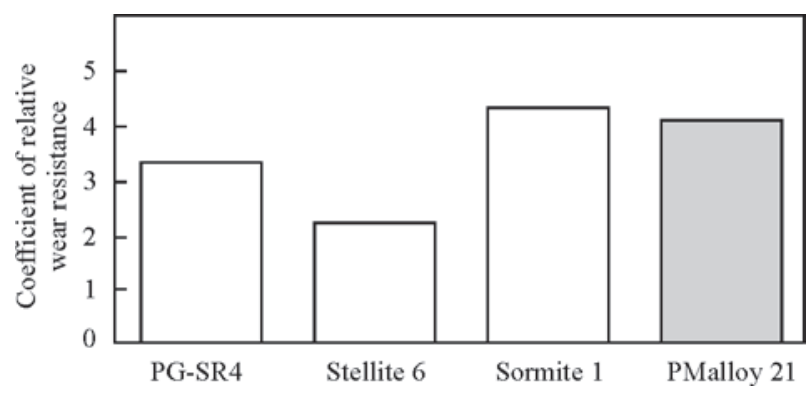

Figure 8. Relative wear resistance of tested commercial alloys as the base metal for manufacture of the screw conveyors were also given.

As can be seen from the Table, CLE of the alloy in as-surfaced state and after heat treatment are significantly different, which is connected with a large difference in the amount of residual austenite. Before heat treatment, it is significantly higher. After tempering at $600{ }^{\circ} \mathrm{C}$, due to the decay of residual austenite, the CLE becomes lower than that of steel $40 \mathrm{KhN}$. During heat treatment of deposited parts it should result in redistribution of residual stresses with the formation of compressive stresses in the deposited layer, which, as is known, favorably affects the operability of parts. From this point of view a high tempering is useful.

During restoration of worn-out parts a high tempering may result in unacceptable deformations caused both by difference in CLEs of the deposited and base metals, as well as volume effect of transformations occurring in the deposited metal during tempering. Therefore, in the given case, a medium tempering should be applied at the temperature of $400^{\circ} \mathrm{C}$, at which structural transformations in the deposited metal are absent.

Experience of industrial application of the alloy. The alloy developed as a powder is used in industry for surfacing screw conveyors by the company «Plasma-Master Ltd» predominantly in Ukraine. For more than 20-year period a wide experience was gained on surfacing and service of deposited parts both in processing of simple as well as filled polymers. At the present time the alloy is produced under trademark PMalloy 21.

In practice, the screw conveyors of casting and extrusion equipment of diameter from 32 to $250 \mathrm{~mm}$ and length from 600 to $5000 \mathrm{~mm}$ are surfaced. Powder PMalloy 21 provides a good formation of deposited metal (Figure 9) and a complete absence of cracks in it, even on very massive parts, at a correct selection of surfacing modes. This important technological advan-

\begin{tabular}{|c|c|c|c|c|c|c|c|c|c|c|c|c|}
\hline \multirow{2}{*}{ Grade of powder (alloy) } & \multicolumn{11}{|c|}{ Content of elements, wt. $\%$} & \multirow{2}{*}{$\begin{array}{c}\text { Hardness } \\
\text { HRC }\end{array}$} \\
\hline & $\mathrm{C}$ & $\mathrm{Si}$ & $\mathrm{Mn}$ & B & $\mathrm{Cr}$ & $\mathrm{V}$ & $\mathrm{W}$ & Mo & $\mathrm{Ni}$ & Co & $\mathrm{Fe}$ & \\
\hline PG-SR4 (Kh15N75S4R4) & 0.7 & 3.9 & - & 3.2 & 15.8 & - & - & - & Res. & - & $\leq 3$ & 58 \\
\hline PG-10K-02 (Stellite 6) & 1.1 & 2.1 & - & - & 28.1 & - & 4.4 & - & - & Res. & - & 42 \\
\hline PG-S1 (Sormite 1) & 2.7 & 2.9 & 2.7 & - & 28.6 & - & - & - & 4.3 & - & Res. & 46 \\
\hline PR-Kh18FNM (PMalloy 21) & 2.2 & 0.8 & 0.9 & - & 18.2 & 7.2 & - & 2.2 & 2.6 & - & Res. & 43 \\
\hline
\end{tabular}


Table 2. Comparison of coefficients of linear expansion depending on heating temperature

\begin{tabular}{|c|c|c|c|c|c|c|c|c|}
\hline \multirow{2}{*}{ Material } & \multirow{2}{*}{ State of alloy } & \multicolumn{7}{c|}{ CLE in the temperature range from $20^{\circ} \mathrm{C}$ to } \\
\cline { 3 - 9 } & After surfacing & 100 & 200 & 300 & 400 & 500 & 600 & 700 \\
\hline $\begin{array}{c}\text { Alloy PR-Kh18FNM } \\
\text { (PMalloy21) }\end{array}$ & After tempering $\left(600^{\circ} \mathrm{C}, 1 \mathrm{~h}\right)$ & 10.5 & 13.6 & 14.0 & 14.1 & 14.7 & 15.0 & - \\
\hline Steel $40 \mathrm{KhN}$ & Initial & 11.8 & 10.7 & 10.8 & 11.0 & 11.5 & 11.8 & 11.2 \\
\hline
\end{tabular}

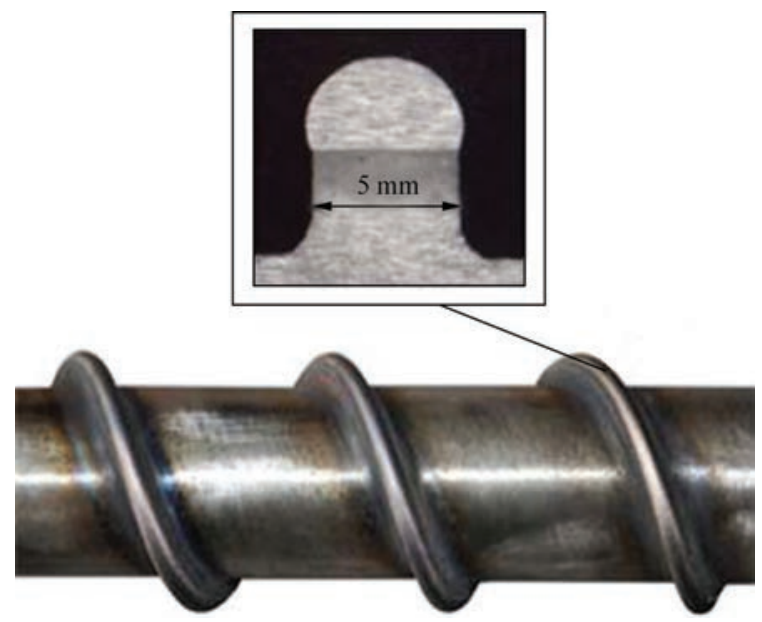

Figure 9. Appearance and macrosection of coil of deposited screw conveyor of $63 \mathrm{~mm}$ diameter

tage of the given alloy allows refusing from pre-heating of the workpiece and, thus, significant simplifying and cheapening the process of surfacing. The powder can be successfully applied both in manufacture of new screws as well as in restoration of worn-out parts. In the latter case, due to a good formation of deposited metal the machining is reduced only to the finish grinding of coil comb in the diameter.

As compared to the nitrated screws the resistance of the deposited ones grew in 3-5 times depending on the type of the processed materials. In Figure 10 the appearance of two screw conveyors of injection molding machine «Kuasy» is shown after service under the same conditions during processing of filled polyamide 6.6.

The photos convincingly prove the advantage of screws, deposited using alloy PMalloy 21. In the first case, the wear of coils is so great that almost nothing left from them, and in the second case they were preserved completely. Despite of some wear of the side surfaces of coils and dents, the screw remains suitable for the further operation and is able to provide a rated efficiency of processing polymers.

At the present time alloy PMalloy 21 is successfully used also for surfacing of other parts like blades of mills for processing of secondary polymers, plungers of hydraulic systems, axles, shafts, etc.

\section{Conclusion}

The complex investigations of structure and properties of developed alloy PMalloy 21 and a long experience of operation of deposited screw conveyors

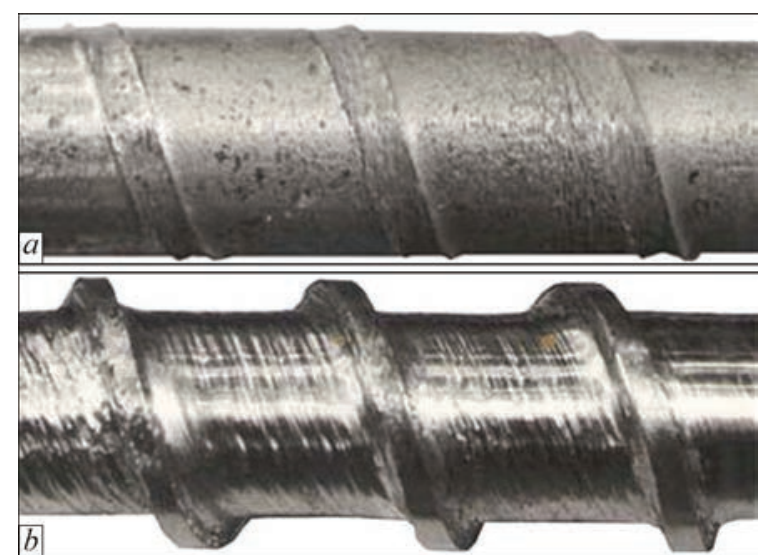

Figure 10. Appearance of screw conveyors of $45 \mathrm{~mm}$ diameter after service during processing of polyamide: $a$ - nitrated screw without surfacing; $b$ - screw deposited using alloy PMalloy 21 with subsequent nitration

convincingly show that the given alloy according to its operability can successfully replace the Ni- and Co-based alloys, traditionally used in industry for surfacing these parts. It is more wear resistant, more technological and considerably cheaper.

1. Maksimchuk, A.M., Mesyats, G.A., Nechiporenko, V.G. et al. (1973) Manufacturing of new and plasma surfacing repair of worn extruder worms. Khimich. i Neft. Mashinostroenie, 8, 22-23.

2. Luelsdorf, P. (1975) Verschleissprobleme mit Zylinder und Schnecke beim Extrudieren. Reilloy-Bericht., 4, 1-8.

3. (1977) Plasma arc weld surfacing - new route to hardfacing screws. Plastics Techn., 23(10), 17-19.

4. Gorka, J., Czuprynski, A., Kik, T. et al. (2011) Przemyslowe aplikacje napawania plazmowego proszkowego. Przeglad Spawalnictwa, 9, 87-94.

5. Gladky, P.V., Pereplyotchikov, E.F., Ryabtsev, I.A. (2007) Plasma surfacing. Kiev: Ekotekhnologiya.

6. Frumin, I.I., Som, A.I., Gladky, P.V. (1981) Plasma surfacing of extruder worms of polymer machines. In: Theoretical and technological principles of surfacing. Surfacing in machine-building and repair: Transact., 13-21. Kiev: PWI.

7. Som, A.I. (1983) New wear-resistant alloy for plasma surfacing. In: Theoretical and technological principles of surfacing. Surfacing consumables: Transact., 7-11. Kiev: PWI.

8. Yuzvenko, Yu.A., Gavrish, V.A., Marienko, V.Yu. (1979) Laboratory units for evaluation of wear resistance of deposited metal. In: Theoretical and technological principles of surfacing. Properties and tests of deposited metal: Transact., 23-27. Kiev: PWI.

9. Poznyak, L.A., Skripchenko, Yu.M., Timaev, S.I. (1980) Die steels. Moscow: Metallurgiya.

10. Popov, V.S., Titukh, Yu.I. (1975) X-ray structural examination of transformations in work surface of alloys under abrasive wear. MiTOM, 1, 24-27. 